\title{
Accidental Corneal Intrastromal Intraocular Lens Implantation with the Wound-Assisted Technique and Clinical Course with Anterior Segment Optical Coherence Tomography
}

\author{
Jo Moriya Shinichi Sakamoto Satoru Inoda Hidenori Takahashi \\ Hidetoshi Kawashima \\ Department of Ophthalmology, Jichi Medical University, Tochigi, Japan
}

Keywords

Wound-assisted technique - Corneal intrastromal implantation - Phacoemulsification .

Anterior optical coherence tomography

\begin{abstract}
Accidental intraocular lens (IOL) implantation into the corneal stroma is a rare clinical entity that can occur during the wound-assisted technique. In this report, we describe a case of an 81-year-old man who underwent cataract surgery in which the IOL was implanted into the corneal stroma with the wound-assisted technique, and we present changes in anterior segment optical coherence tomography. The IOL was removed and reinserted after widening the incision. Air tamponade was created by intracameral injection. An anterior chamber tap was performed $10 \mathrm{~h}$ later to reduce increased intraocular pressure. Interlayer separation of the corneal stroma was confirmed 30 min postoperatively but was corrected 4 days later. The patient's best-corrected visual acuity (BCVA) was logMAR 0.30, and he had been diagnosed with age-related macular degeneration before surgery. Although the opacity of the corneal stroma persisted, BCVA improved to logMAR 0 . When using the wound-assisted technique for IOL insertion, surgeons should take care not to implant the IOL into the corneal stroma.
\end{abstract}

(C) 2021 The Author(s).

Published by S. Karger AG, Basel

\section{Introduction}

Cataract surgery is now being performed through smaller incisions with many different types of injectors and foldable intraocular lenses (IOLs). The wound-assisted technique is one of several methods that facilitate IOL implantation through a smaller incision. By using this 
Moriya et al.: Corneal Intrastromal IOL Implantation with the Wound-Assisted Technique

technique, a rolled IOL can be implanted directly through the incision with an injector without inserting the cartridge tip $[1,2]$.

Although there have been 4 case reports of corneal intrastromal IOL implantation with this technique [3-7], to our knowledge, no similar reports have included anterior segment optical coherence tomography (AS-OCT) findings. In this report, we describe the long-term course of accidental corneal intrastromal IOL implantation with AS-OCT findings.

\section{Case Report}

An 81-year-old man was referred to our hospital for cataract surgery. He had undergone intravitreal injection of anti-vascular endothelial growth factor 19 times over the course of 7 years for treatment of neovascular age-related macular degeneration before the current surgery. He underwent cataract surgery 5 months after the last intravitreal injection.

Best-corrected visual acuity (BCVA) was logMAR 0.30 (20/40). Intraocular pressure (IOP) was within normal limits, and endothelial cell density (ECD) was 2,817 cells $/ \mathrm{mm}^{2}$. The wound-assisted technique was used for IOL implantation. A temporal corneal incision 2.4 $\mathrm{mm}$ ) was made with a 2.4-mm-wide disposable stainless steel knife (Mani, Inc., Tochigi, Japan) at once, instead of multilayer and stepped incision. Phacoemulsification and aspiration was performed using a phacoemulsification system (INFINITI ${ }^{\circledR}$; Alcon, Geneva, Switzerland). The surgical procedure was performed without any problem until the actual IOL insertion step. The IOL was inserted by allowing the leading haptic to protrude from the cartridge tip and subsequently trying to inject the 6-mm IOL (AcrySof ${ }^{\circledR}$ IQ Toric SN6AT3 17.5 diopter; Alcon, Geneva, Switzerland) with the Royale III injector (ASICO, Westmont, IL, USA) and the Monarch $^{\circledR}$ III D cartridge (Alcon). When we attempted to perform IOL injection using the wound-assisted technique, we encountered strong resistance that the eyeball markedly rotated nasally. The resistance persisted, so injection was aborted. We then discovered the IOL had been accidentally implanted into the corneal stroma (Fig. 1a; online suppl. Video 1; for all online suppl. material, see www.karger.com/doi/10.1159/000514068). We unsuccessfully attempted to remove the IOL with micro-corneal suturing forceps (Inami, Tokyo, Japan). The IOL was eventually removed with German nontoothed forceps (Inami), and subsequently, the anterior chamber (AC) was refilled with viscoelastic substance. We did not wash out the viscoelastic substance in the intrastromal gap. The incision was widened to a width of $2.5 \mathrm{~mm}$, and the IOL was inserted after confirming that the cartridge tip was in the $\mathrm{AC}$. The procedure was completed by filling the $\mathrm{AC}$ with air. The patient was instructed to rest in the supine position after the surgery.

At 30 min postoperatively, opacity, edema, and interlayer separation were confirmed using AS-OCT (CASIA2; Tomey Corporation, Nagoya, Japan) imaging of the corneal stroma, where the IOL had been accidentally inserted (Fig. 1b). Then, $10 \mathrm{~h}$ postoperatively, the patient complained of eye pain, and severe corneal edema with an increased IOP of $60 \mathrm{~mm} \mathrm{Hg}$ was noted. AC tap reduced the IOP to $19 \mathrm{~mm} \mathrm{Hg}$. The next morning, interlayer separation was found not to have worsened, and he was started on $0.1 \%$ betamethasone sodium phosphate (Sanbetason ${ }^{\circledR}$; Santen Pharmaceutical Co., Ltd., Osaka, Japan) and 0.5\% levofloxacin (Levofloxacin Hydrate ${ }^{\circledR}$; Pfizer Japan Inc., Tokyo, Japan) eye drops 4 times daily.

Four days postoperatively, corneal opacity persisted, but AS-OCT showed some improvement of the edema and interlayer separation (Fig. 1c). ECD was 2,941/ $\mathrm{mm}^{2}$. As of 1 week after the procedure, BCVA was logMAR 0.52 (20/63), ECD had not changed much, and the corneal opacity was gradually subsiding.

Two weeks postoperatively, he received intravitreal injection of ranibizumab for subretinal pigment epithelium hemorrhage due to neovascular age-related macular degeneration. BCVA

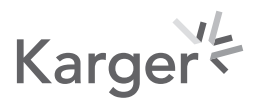



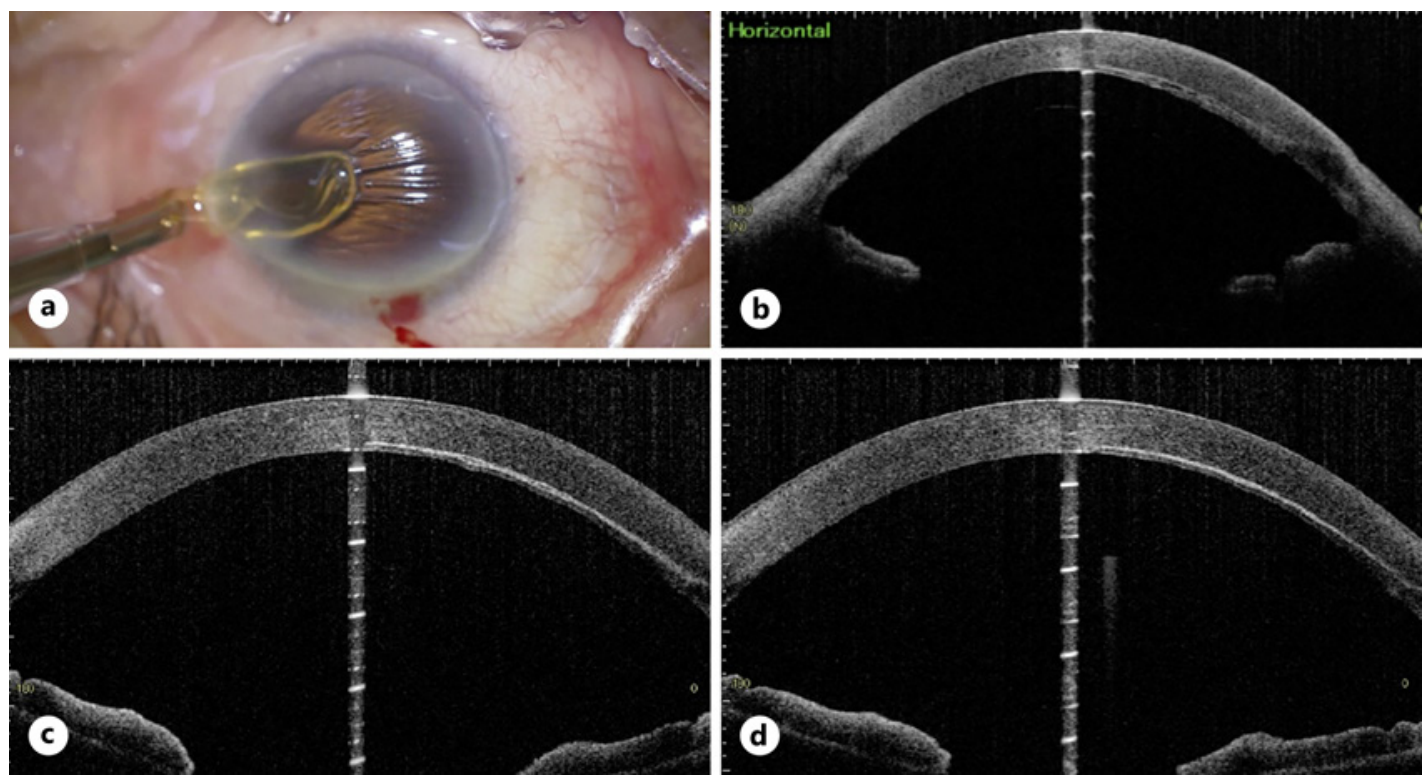

Fig. 1. Corneal intrastromal IOL implantation with the wound-assisted technique during phacoemulsification and clinical course after corneal intrastromal IOL implantation with AS-OCT. a Intraoperative photograph. Corneal fold due to the IOL is seen. b AS-OCT $30 \mathrm{~min}$ postoperatively. Intercalation of the deep corneal stroma is observed. c AS-OCT 1 month postoperatively. Deep corneal stromal opacity is observed. d A slit-lamp photograph taken 2 months postoperatively. Corneal opacity is reduced. IOL, intraocular lens; AS-OCT, anterior segment optical coherence tomography.

improved to $\operatorname{logMAR} 0.22(20 / 33)$ at 1 month postoperatively and to $\log$ MAR $0.19(20 / 31)$ at 2 months afterward. Corneal opacity persisted, but its size had decreased. BCVA improved to $\log$ MAR $0(20 / 20)$ at 4 months postoperatively with minimal corneal opacity (Fig. $1 \mathrm{~d})$. The number of instillations of betamethasone sodium phosphate eye drops was gradually decreased from 4 to 2 after 2 to 4 months of the procedure.

\section{Discussion/Conclusion}

In this case, the IOL was accidentally implanted into the corneal intrastromal space, but BCVA improved to logMAR 0 postoperatively. The only complication was increased IOP postoperatively due to intracameral air injection.

The pressure that initially caused the interlayer separation of the cornea was likely due to insertion of the injector tip, which is typically inserted with the bevel down. In this case, when IOL insertion was attempted, the eye was markedly rotated nasally.

An instructional course conducted by the American Society of Cataract and Refractive Surgery noted the importance of fixing the eye in the straight position with both hands before IOL insertion and then to insert the IOL under direct vision to prevent corneal intrastromal implantation [5]. However, if the eye is pushed with a strong force and rotated as in our case, eye fixation from the opposite side is impossible. In addition, because subconjunctival hemorrhage occurs at a high rate when grasping and fixing the eyeball, inserting the tip with a force that does not require fixation may prevent corneal intrastromal implantation and subconjunctival hemorrhage.

The onset of the interlayer separation of the cornea may be related to cartridge tip sharpness. We used the HOYA Vivinex ${ }^{\mathrm{TM}}$ iSert $^{\circledR}$ and KOWA Avansee ${ }^{\mathrm{TM}}$ inserts much more than AcrySof. These have sharper edges than AcrySof, and they cannot be used for the wound-assisted 
Moriya et al.: Corneal Intrastromal IOL Implantation with the Wound-Assisted Technique

technique. Although not used in this case, the multilayer and stepped incision is also considered to be one of the risks. Because the IOL diopter was not excessively high, the IOL diopter might not be a factor associated with the corneal separation in this case.

Air injection into the $\mathrm{AC}$ is used for treatment of Descemet's membrane detachment. This procedure is recommended in cases that involve Descemet's membrane detachment with a gap $>1 \mathrm{~mm}$ [8]. After air injection, the patient was required to rest in the supine position with careful monitoring of IOP. AS-OCT offers good visualization of postoperative changes in the cornea. A previous report using AS-OCT for longitudinal corneal wound evaluation after phacoemulsification surgery found Descemet's membrane detachment was present in $37.1 \%$ of cases within 1 day postoperatively, persisted 1-3 months postoperatively in $4.5 \%$, and was absent $>3$ months postoperatively in $100 \%$ [9]. In our case, interlayer separation might have improved earlier if we had washed the viscoelastic substance out of the interlayer.

It is important to consider the possibility of corneal intrastromal IOL implantation during IOL implantation with the wound-assisted technique. If some surgeons encounter excessive resistance while injecting an IOL, they should stop injecting immediately and investigate the cause.

\section{Acknowledgement}

We thank Dr. Kasuya, Dr. R. Takahashi, and Dr. Arai for their treatment of the patient. This paper is derived from details presented at the 34th Annual Meeting of the Japanese Society of Cataract and Refractive Surgery, Kyoto, Japan, in October 2016.

\section{Statement of Ethics}

The patient described in this study provided written informed consent to publish his case. The study protocol was approved by the IRB of Jichi Medical University (CU19-132).

\section{Conflict of Interest Statement}

Dr. Takahashi received lecturer's fees from Kowa Pharmaceutical, Novartis Pharmaceuticals, Bayer Yakuhin, and Santen Pharmaceuticals, and grants from Bayer Yakuhin and Novartis Pharmaceuticals, outside this work. Dr. Kawashima received lecturer's fees from Kowa Pharmaceutical, Novartis Pharmaceuticals, and Santen Pharmaceuticals outside this work. Dr. Moriya, Dr Sakamoto, and Dr. Inoda declare no potential conflict of interest.

\section{Funding Sources}

This research did not receive any specific grant from funding agencies in the public, commercial, or not-for-profit sectors.

\section{Author Contributions}

J.M. acquired data and drafted the manuscript. S.S. and S.I. analyzed data and revised the manuscript. H.T. designed the study and revised the manuscript. H.K. analyzed data, revised the manuscript, and supervised the study.

\section{Karger'}


Moriya et al.: Corneal Intrastromal IOL Implantation with the Wound-Assisted Technique

\section{References}

1 Tsuneoka H, Hayama A, Takahama M. Ultrasmall-incision bimanual phacoemulsification and AcrySof SA30AL implantation through a $2.2 \mathrm{~mm}$ incision. J Cataract Refract Surg. 2003 Jun;29(6):1070-6.

2 Shiba T, Tsuneoka H. Prewound assisted technique for hydrophobic foldable intraocular lens implantation. Eur J Ophthalmol. 2017 Aug;27(5):569-72.

3 Hogden MC, Maccheron LJ, Beckingsale PS. Intracorneal intraocular lens injection. Clin Exp Ophthalmol. 2008 Mar;36(2):191-2.

4 Jain AK, Gupta A, Gupta R, Nawani N. Inadvertent corneal intrastromal intraocular lens implantation during phacoemulsification. JAMA Ophthalmol. 2014 Jun;132(6):713.

5 Jain AK. Accidental corneal intrastromal implantation of intraocular lens with wound-assisted docking technique during phacoemulsification and its management. [cited 2019 Aug 4] Available from: http://ascrs.org/ center-for-learning/video/accidental-corneal-intrastromal-implantation-intraocular-lens-wound-assisteddocking-technique.

6 Lok JY, Young AL. Corneal injection track: an unusual complication of intraocular lens implantation and review. Int J Ophthalmol. 2015 Jun;8(3):631-3.

7 Meiklejohn BD, O’Hagan S. Unexpected intrastromal insertion of square-edged intraocular lens during routine cataract surgery. J Ophthalmol Vis Neurosci. 2018 Dec;3(3):26.

8 Mahmood MA, Teichmann KD, Tomey KF, Al-Rashed D. Detachment of Descemet's membrane. J Cataract Refract Surg. 1998 Jun;24(6):827-33.

9 Wang L, Dixit L, Weikert MP, Jenkins RB, Koch DD. Healing changes in clear corneal cataract incisions evaluated using Fourier-domain optical coherence tomography. J Cataract Refract Surg. 2012;38(4):660-65. 